\title{
Thermophysics for Manufacturing Engineers: A Nontraditional Approach
}

\author{
Mark Schumack, Michael Vaksman, Shuvra Das, Kevin Belfield \\ Mukasa Ssemakula, Roger Pryor, Vladimir Sheyman \\ William W. Schultz \\ University of Detroit Mercy/Wayne State University/University of Michigan
}

\begin{abstract}
This paper describes the development of a set of instructional modules that will introduce candidates to the principles of thermal/fluid sciences, with applications to manufacturing engineering. The candidates are currently employed as machinists at the Center for Advanced Technologies (CAT), a world-class manufacturing facility which is part of the civil rights organization Focus:HOPE in Detroit. The Greenfield Coalition is a collaboration among six universities to develop a learner-centered, experiential-based, team-oriented, interdisciplinary curriculum for manufacturing engineering which will be launched at the CAT and later transferred to legacy university programs.
\end{abstract}

Thermal/fluid sciences (thermodynamics, fluid mechanics, and heat transfer) are typically considered to be among the most difficult of topics taught in an engineering curriculum. This project makes the subject matter more accessible to candidates by embedding the material within the context of manufacturing activities that are part of the candidates' daily experience. The foundational principles for most of the eighteen modules are taught in interactive tutorials written with the multimedia authoring package Authorware ${ }^{\circledR}$. Complementing the computer-based tutorials are instructor-assisted problem-solving sessions, experiments, work-related team projects, case studies, or traditional lectures. The tutorials include periodic assignments such as computer problems (solved using a spreadsheet or mathematics package) and traditional "penciland-paper" problems.

Presented here is a discussion of the teaching philosophy, a description of the structure of the thermophysics curriculum, and a summary of content. Portions of the computer-based tutorials are described, and examples of experiments, projects, and case studies are given.

\section{INTRODUCTION}

Traditionally, thermodynamics, fluid mechanics, and heat transfer are difficult subjects for students to master. Thermodynamics in some universities, for example, has failure rates as large as $20 \%-40 \%$, contributing to poor retention rates in engineering [1]. Students eager to learn at the beginning of the term in fluid mechanics soon become lost as they struggle with the calculus and identification of appropriate models for various flow phenomena. By the time they reach heat transfer (if they get that far) - a subject that requires knowledge of both thermodynamics and fluid mechanics - many have resigned themselves to an insecure understanding at best, and 
concentrate their efforts on other classes that deal with more concrete subjects.

A number of textbooks have dealt admirably with the presentation of thermal science material. Moran and Shapiro [2] is an introductory thermodynamics text that most students at the University of Detroit Mercy admit gives clear explanations. The text also offers a large selection of open-ended problems that give students exposure to more "real-world" problems. Incropera and DeWitt [3] is a heat transfer text that boasts a host of practical examples and exercises covering a wide range of applications. In spite of the availability of good textbooks, however, students still have a hard time applying general concepts such as the conservation of energy, the momentum equation, or the second law to specific situations. Students struggle over simply comprehending the material, let alone using the principles to analyze or design systems.

The NSF-sponsored Greenfield coalition offers an excellent opportunity to explore nontraditional methods for presenting thermophysics. (The traditional method is taken here to be the lecture). The instructional material is presented at the Center for Advanced Technologies (CAT), a manufacturing facility in Detroit employing a large minority population, and the center for an Associate's and Bachelor's level Manufacturing Engineering degree program. Students in the program (called "candidates" at the facility) are all full-time machinists. They receive onsite instruction after their work shifts in the form of lectures, computer-based tutorials, and hands-on laboratory experiences.

The objective of this effort is to teach the fundamentals of thermodynamics, fluid mechanics, and heat transfer within a manufacturing context, with particular applications in the CAT. Thermalfluid phenomena are ubiquitous in manufacturing activities. Examples include the delivery of cutting fluids to workpiece/tool interfaces, the heat flow and related temperature profiles in cutting operations, the solidification of castings, the fluid jet cooling of steel strips emerging from rolling mills, quenching, furnace drying and surface-treatment operations, manufacture of polymer filaments, welding, plasma spray coating, laser shaping, and thermal treatment of semiconductors. The importance of understanding thermal phenomena is crucial to the control of parameters such as surface finish, residual stress, fatigue strength, hardness, and part tolerances. At the CAT, there are a host of other non-manufacturing-related phenomena, such as operation of the climate control system, performance of a cogeneration facility for electricity and steam, and characteristics of the building water supply system.

The curriculum development capitalizes on the principle that students grasp principles most effectively if the principles are presented first in the context of familiar phenomena and then generalized. Accordingly, candidates will learn how to apply governing equations through exposure to a wide variety of examples from their spheres of experience. The material will be presented in a series of 18 modules developed by thermal science instructors from the University of Detroit Mercy, Wayne State University, and the University of Michigan. The Thermophysics courseware development is one of the three stems constituting the Engineering Physics knowledge area for the Greenfield Coalition. The other two stems are Mechanophysics and Electrophysics. Initial delivery of the thermophysics material is slated for January of 1997. 


\section{APPROACH}

Computer-based presentations form the foundation of the delivery and are written with the multimedia package Authorware ${ }^{\circledR}$. Recognizing the drawbacks to a curriculum delivered solely on the computer screen, other types of activities are incorporated to satisfy a variety of learning styles. Examples include hands-on laboratories, team design and analysis projects, instructor-led problem-solving sessions, and lectures.

The first five modules (developed by M. Ssemakula and R. Pryor of Wayne State University) consist of the subjects normally found in a first- or second-year engineering physics course, covering fluids, thermal properties, the first and second laws of thermodynamics, and the kinetic theory of ideal gases. The primary form of instruction consists of laboratory exercises. Each experiment is preceded by a computer tutorial written in Labview ${ }^{\circledR}$ which introduces the candidate to the background material, provides an application usually in the manufacturing context, assigns reading and homework problems, and demonstrates the experiment via animation. The modular experiments fit on table tops and are located at the CAT. Grading for these five modules is based on homework sets and group laboratory reports. There are also individual (pencil-and-paper) tests at the end of each module.

The remaining thirteen modules, developed by M. Schumack, M. Vaksman, S. Das, and K. Belfield at the University of Detroit Mercy, W.W. Schultz at the University of Michigan, and V. Sheyman at Wayne State University, cover the foundational material more thoroughly, and introduce thermal science topics tailored to a manufacturing audience. The primary delivery method is computer-based tutorial, augmented by other teaching approaches mentioned above and described in more detail below. Grades are mostly determined based on homework solutions, project reports, and final exams given at the end of each module. Testing is done via handouts in a traditional exam setting (with two exceptions) to minimize the possibility of cheating that could occur if candidates were taking tests at their own convenience on the computer.

One danger that threatens a time-pressured faculty developer is the temptation to produce an electronic textbook. In this rudimentary form of a computer tutorial, the only interaction is the user's pressing of a button to move to the next screen of text. Our effort, with the help of student programmers, is to include as many capabilities as modern computers and software packages allow. These capabilities fall into two categories: presentation and interaction. Presentation capabilities include video and audio clips, animations, color figures and drawings, and photographs. Interaction capabilities include quizzes that provide instant feedback and redirection to relevant sections of the module if necessary, hypertext links, access to reference material from anywhere in the module, and user-directed simulations that allow parametric investigations. Quiz questions are presented frequently throughout a module to gage the candidate's progress, and take the form of multiple choice, drag and drop, text entry, and "hot spot" types of interrogation. 
The series of eighteen modules has been divided into five one-credit hour courses. The modular breakdown can be seen in figure 1. The estimated number of hours that a candidate actually spends interacting with the module vehicle (computer, laboratory, problem-solving session, lecture) varies from 4 to 14 hours. These times are largely speculation in this pre-delivery period.

The candidates are not on campus and therefore miss the convenience of instructors' office hours. To compensate for this, candidates are provided with instructors office phone numbers and email addresses. The syllabus typically states the times and days the instructor is in his office. In a current preliminary offering of the first five modules, M. Schumack also maintains a web page with hints on homework problems (see http://www.udmercy.edu/htmls/personal/schumamr/schumamr.htm).

A single textbook covering all the material taught here is commercially unavailable. It was felt, however, that the candidates would appreciate the security of something that they could take away from the course other than a few graded assignments and the memory of a multitude of computer screens. We subsequently chose Fundamentals of Physics by Resnick et al. [4] for the first five modules and Introduction to Thermal Sciences by Wolgemuth et al. [5] for the remaining modules. Candidates are anchored to these texts through occasional reading and problem assignments, in addition to the computer tutorials.

\section{MODULE DESCRIPTIONS}

The eighteen modules are described below. Examples of projects, experiments, and case studies are given where appropriate. The first five modules are developed by M. Ssemakula (Engineering Technology) and R. Pryor (Institute for Manufacturing Research) of Wayne State University. Developers for the remaining modules are cited in the descriptions.

Fluid Properties This modules covers pressure and variation with depth, viscosity, surface tension, Archimedes' principle, specific gravity, and the Bernoulli equation. Two experiments in this module demonstrate the variation of pressure with fluid depth and Archimedes' principle.

Thermal Properties Topics covered in this module include temperature, temperature scales, thermal expansion, coefficient of expansion, specific heat, change of state, and latent heat. Two experiments are performed: a demonstration of the thermal expansion characteristics utilizing the metrology equipment at the CAT, and an illustration of the zeroth law and its use in measuring temperature along with exposure to different temperature scales.

Introduction to First Law of Thermodynamics The mechanical equivalent of heat, work, first law of thermodynamics, internal energy, and thermodynamic processes are the topics covered in the third module. One experiment demonstrates the conversion of mechanical energy into heat (friction). A second experiment demonstrates the specific heat capacity of water and heat of 
transformation of ice-water.

Introduction to Second Law of Thermodynamics Topics covered in this module are heat engines, second law, reversible and irreversible processes, Carnot cycle, examples of real heat engines, heat pumps, and refrigerators.

Kinetic Theory of an Ideal Gas This module covers the equation of state of an ideal gas, molecular model for pressure of an ideal gas, relationship of temperature and molecular kinetic energy, specific heat of an ideal gas, and the Maxwell-Boltzmann distribution of speeds. An experiment demonstrates the change in temperature of a compressed gas.

Chemistry and Cutting Fluids This computer-based tutorial, written by K. Belfield in the Chemistry Department at UDM, covers the fundamental principles of both chemical and physical properties of cutting fluids. The module begins with a review of chemical fundamentals. Physical changes of properties of fluids such as viscosity and density due to temperature variations are detailed. Chemical changes, such as oxidative degradation, are also covered. Discussion of petroleum-based and synthetic fluids highlights basic organic chemistry. Candidates also learn about selecting lubricants and cutting fluids through a collaborative assignment and report. The candidates are commissioned to find out what type of cutting fluid is used in machining operations at the CAT, describe why this particular fluid is used, and summarize the physical properties.

Thermodynamics and the First Law, Entropy and Heat Engines, and Real Gases These three modules, produced by M. Vaksman from the Chemistry Department at UDM, explore material typically taught in a junior-level thermodynamics course. Upon completion of the tutorial, the candidate will be able to estimate the amounts of heat evolved and work done in the model processes related to manufacturing. A video clip of an experiment designed to show the energy partition in a drilling operation is included, along with animations showing the molecular behavior in grinding and internal combustion engine operation.

Hydrostatics and Hydraulics The topics covered in this module, developed by S. Das from the Mechanical Engineering Department at UDM, include pressure/depth relations, Archimedes' principle, and hydraulic levers. The computer-based tutorial is complemented by problemsolving sessions (instructor-assisted) and a case study in which candidates analyze a hydraulicpower device similar to that found on the fixture mechanisms in modern machining consoles.

Internal Fluid Flow The topics covered in this module, developed by W.W. Schultz at UM, include laminar flow relations, the energy equation for pipe flow, pump relations, and an introduction to lubrication phenomena. The computer-based tutorial will be complemented by a team project involving the re-design of a cutting fluid delivery system in order to increase the flow rate. This project familiarizes the candidates with the basic relationships for flow friction losses and pump power. 
Conduction Heat Transfer - One Dimensional Steady-State Topics covered in this module, developed by M. Schumack from the Mechanical Engineering Department at UDM, include thermal conductivity, Fourier's Law of Conduction, Newton's Law of Cooling, and the onedimensional heat equation. The computer-based tutorial includes examples and problems dealing with grinding, drilling, and analysis of heat loss through insulated piping in the CAT cogeneration facility. Candidates analyze the heat flow in a grinding operation using published experimental data in a problem-solving session coached by the instructor. Figure 2 shows a screen from the module.

Conduction Heat Transfer - Transient Topics covered in this module, developed by S. Das and M. Schumack, include the unsteady lumped formulation and an introduction to onedimensional unsteady conduction. The computer-based tutorial is complemented by a case study of parts that arrive on the shop floor at either very cold or hot temperatures which would result in out-of-tolerance parts if machined without first allowing the parts to reach ambient temperature. The case study will be presented as an open-ended problem for which the candidates will determine an estimated "cool-down" period and provide a written discussion.

Convection Heat Transfer Nusselt number correlations for external, internal, and natural convection flows are covered in this module, developed by M. Schumack. Dimensional analysis is introduced through a derivation of the dimensionless parameters for the temperature rise in a metal cutting operation. In addition to the homework problems required as part of the computerbased tutorial, problems where the candidates must calculate heat transfer coefficients for various geometries found in the CAT environment will be presented in instructor-assisted problemsolving sessions.

Heat Exchangers Topics covered in this module, developed by M. Schumack, include energy balance equations for heat exchangers; the log-mean temperature difference equation; concentric tube, shell-and-tube, and compact heat exchangers. The computer-based tutorial will be complemented by candidates' use of a computer analysis package to perform the preliminary design of a heat exchanger to cool cutting fluids. The candidates perform two group design projects for this module. One, based on an actual thermal problem at the CAT, involves the specification and sizing of a heat exchanger to decrease the temperature of the fluid entering the machines. The second team project is a redesign of the CAT air handling system.

Radiation Heat Transfer This module is developed by M. Schumack and includes the StefanBoltzmann law, view factors, and radiation exchange between gray surfaces. The computerbased tutorial will be complemented by an instructor-assisted problem-solving session where principles regarding radiation exchange between surfaces and comparative importance with respect to convection heat transfer are covered.

Thermal Aspects of Manufacturing Processes This module, developed by S. Das, pulls together much of the material from the previous modules to address a number of manufacturing operations. Topics include expansion and contraction of materials and effects on tolerances, 
relations for heat produced during machining operations, and phase-change phenomena in welding. The computer-based tutorial will be complemented by case studies.

Heat Transfer and Fluid Flow in Casting This module will be developed by V. Sheyman from Engineering Technology at WSU. The computer-based tutorial on physical mechanisms in the casting process will be complemented by a three case studies of a mold-flow problem which brings together elements of heat transfer and fluid flow taught in previous modules.

\section{LESSONS LEARNED}

The real test of the effectiveness of our approach will occur during the first module offering in January 1997. The lessons learned to this point have therefore been mainly in the area of multimedia courseware development. A more traditional offering of the material covered in the first five modules was begun in November of 1996, however, and provides some insight into the candidates' learning styles.

The developers took different approaches to actual execution of the modules. Some developers preferred to learn Authorware and do the multimedia authoring themselves, while others wrote the text and interaction instructions and had student assistants do the programming. Because of the time consumed in developing illustrations, animations, and video editing, most of the graphics were done by students. The following list summarizes lessons learned so far.

$\checkmark \quad$ Much time was spent researching the software packages and hardware necessary to produce graphics and sound recordings. A multimedia authoring station should consist of at least the following components: state-of-the-art PC, removable data storage device (multimedia programs are typically 5 to $50 \mathrm{MB}$ ), 2D and 3D drawing package, animation package, video capture card and editing software, and a utility to capture screen images.

- Users should be stimulated with both sound and graphics. Students are used to flesh-andblood teachers. If the student is to learn from the computer, it had better provide an experience at least as colorful as a live performance.

- The production of a multimedia module of the visual and audio quality found in some of the CDs put out by the large publishing houses is unattainable through the efforts of a faculty member and one or two student assistants. If high quality images and animations are required, then a multimedia development team of dedicated professionals is the only route. An entirely satisfactory module, however, can be produced by a faculty member with student help if the many features of current multimedia authoring programs are exploited.

A preliminary offering of the first five modules was started in November 1996. The delivery was mainly in the traditional lecture style, but offered an opportunity to assess the learning styles of the CAT candidates. A learning styles inventory was developed by M. Schumack and V. 
Woodell of the Greenfield Coalition incorporating elements from published tests [6], but tailored to identify four learning style categories: verbal, visual, kinesthetic, and social. The first three popular classifications are discussed elsewhere [see, for instance, 7], but the social learner perhaps needs some explanation. As we define it, a learner who is "social" generally prefers learning through interaction with people as opposed to books, videos, or computer-based tutorials.

Figure 3 shows the class averages for each of the four learning styles. Most candidates favor visual learning while the preference for verbal learning was lowest. This emphasizes the need for multimedia tutorials to be highly graphics-oriented and not simply a procession of flickering text. The social learning style also received a high percentage, indicating that some personal instructor interaction is essential in the delivery process. Our laboratory element, particularly in the first five modules, addresses the preference for kinesthetic learning.

\section{CONCLUSION}

The eighteen modules discussed comprise a five-credit hour offering applicable to both the Associate and Bachelor Degree programs at the CAT. Although the final judgment on the effectiveness will come only after the candidates have been assessed, preliminary reviews by peers and NSF examiners indicate the approach will be successful. Efforts are also underway to incorporate some of the material into traditional campus programs. Animations or threedimensional graphics will be used to present material impossible to draw on a chalkboard. The computer tutorials will be offered in abridged form to students who wish to brush up on the subjects outside of regular class time. Some of the interactive aspects of the tutorials may also be used to enhance dialogue between instructor and students during class time.

This work is funded through a subcontract from the Greenfield Coalition, under NSF Agreement \#EEC-9630951.

\section{REFERENCES}

1. Cobourn, W.G. and Lindauer, G.C., "A Flexible Multimedia Instructional Module for Introductory Thermodynamics,” Journal of Engineering Education, pp. 271-277, July 1994.

2. Moran, M.J. and Shapiro, H.N., Fundamentals of Engineering Thermodynamics, 3rd edition, John Wiley \& Sons, Inc. (1995).

3. Incropera, F.P. and DeWitt, D.P., Introduction to Heat Transfer, 3rd edition, John Wiley \& Sons, Inc. (1996).

4. Halliday, D., Resnick, R., and Walker, J., Fundamentals of Physics, 4th edition, John Wiley \& Sons, Inc. (1993).

5. Schmidt, F.W., Henderson, R.E., and Wolgemuth, C.H., Introduction to Thermal Sciences, 2nd edition, John Wiley \& Sons, Inc. (1993).

6. Kirby, J.R., Moore, P.J., and Schofield, N.J., "Verbal and Visual Learning Styles," Contemporary 
Educational Psychology, vol. 13, pp. 169-184 (1988).

7. $\quad$ Felder, R., "Matters of Style," ASEE Prism, pp. 18-23, December 1996.

\section{BIOGRAPHICAL INFORMATION}

MARK SCHUMACK is Associate Professor of Mechanical Engineering at the University of Detroit Mercy. He received his Ph.D from the University of Michigan in 1991. Prior to obtaining his doctorate, he worked as a design engineer at the Cleveland Electric Illuminating Company.

MICHAEL VAKSMAN is Assistant Professor of Chemistry at the University of Detroit Mercy. He received his $\mathrm{Ph} . \mathrm{D}$ from the School of Chemistry at Tel Aviv University in 1992, and did post-doctoral work at Texas A\&M University.

SHUVRA DAS is Assistant Professor of Mechanical Engineering at the University of Detroit Mercy. He received his Ph.D in Engineering Mechanics from Iowa State University in 1991. After doing post-graduate work, he was an Analysis Engineer at Concurrent Technologies.

KEVIN BELFIELD is Assistant Professor of Chemistry at the University of Detroit Mercy. He received his Ph.D in organic chemistry from Syracuse University in 1988, and did post-doctoral work at SUNY-Syracuse and Harvard University.

MUKASA SSEMAKULA is an Associate Professor in the Division of Engineering Technology at Wayne State University. He received his Ph.D in Mechanical Engineering from the University of Manchester Institute of Science and Technology in 1984.

ROGER PRYOR is Associate Professor (Research) at the Institute for Manufacturing Research at Wayne State University. He has obtained extensive industrial experience since receiving his Ph.D from Pennsylvania State University in 1972.

WILLIAM W. SCHULTZ is Associate Professor of Mechanical Engineering at the University of Michigan. He received his Ph.D in Applied Mathematics and Engineering Sciences from Northwestern University in 1982. Prior to obtaining his Ph.D, he was a Research Engineer at Owens/Corning. 


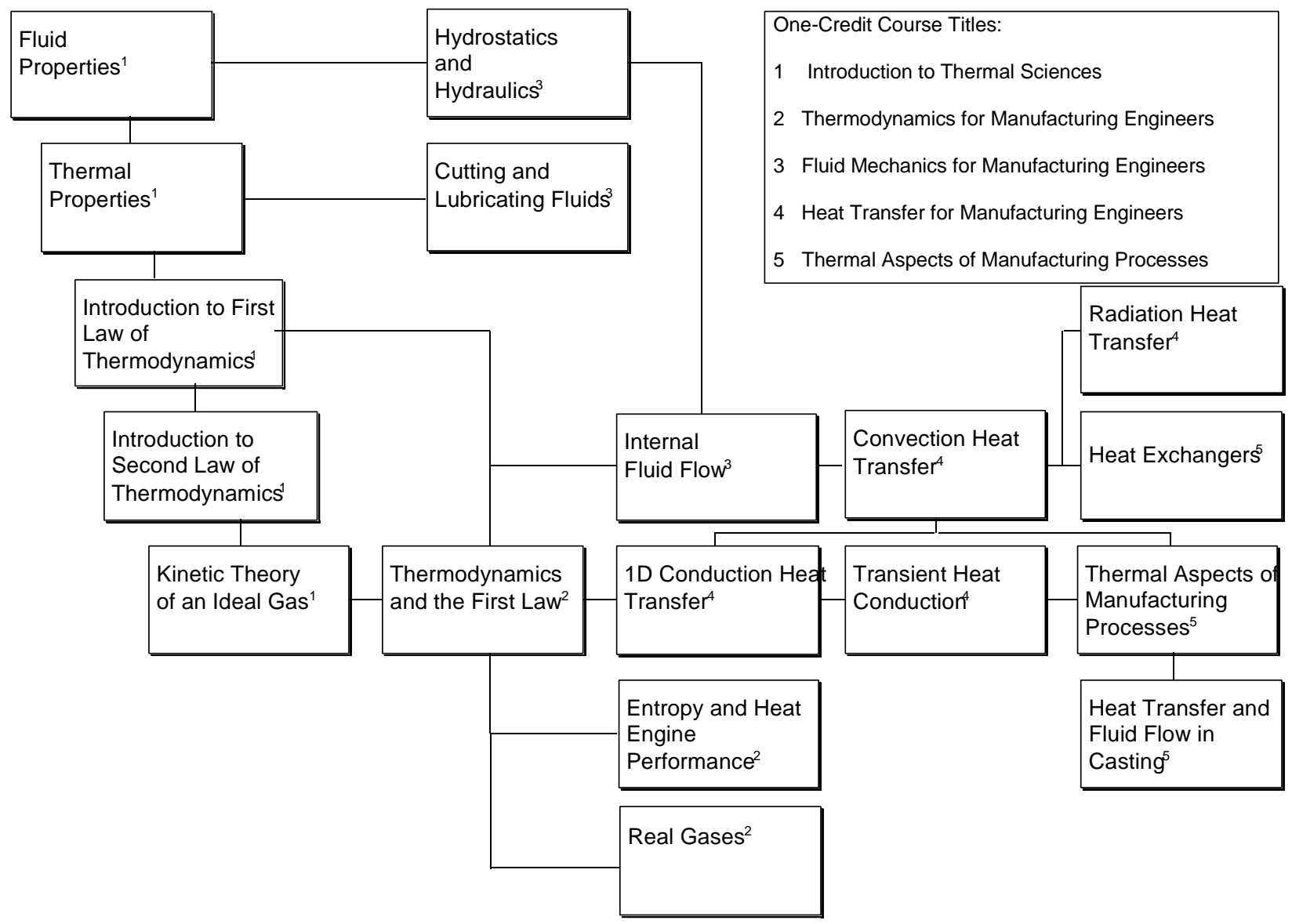

Fig. 1. Flowchart for Thermophysics. Also shown is how the modules are grouped for the onecredit course offerings. 


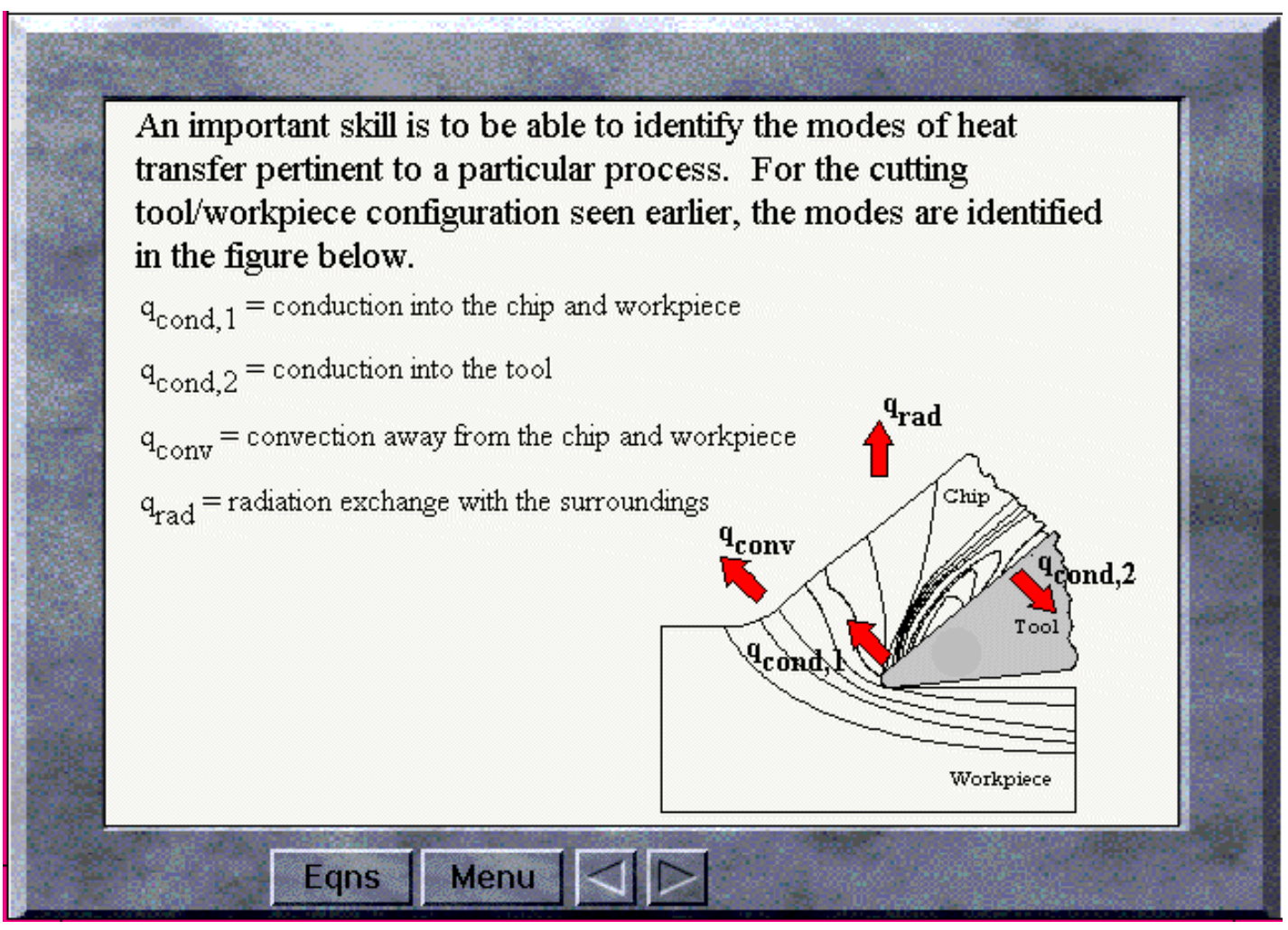

$\mathrm{Fi}$

g. 2 .

Screen from the module "Conduction Heat Transfer - One Dimensional Steady State."

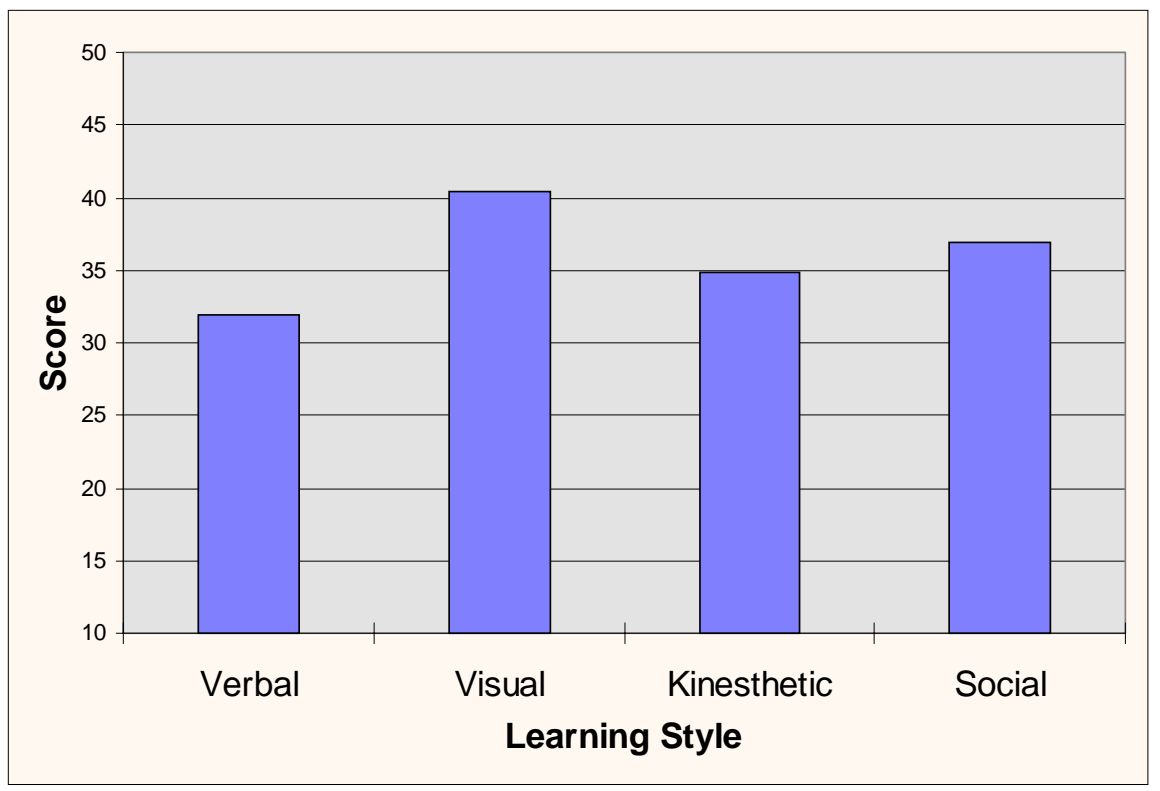

Fig. 3. Average scores for each of the learning styles. The class size was sixteen. The highest and lowest possible scores for each style were 50 and 10 , respectively. 